\title{
Examen clinique objectif structuré (ECOS) formatif avec patient simulé et rétro-action par un pair en visioconférence
}

\section{Formative Objective Structured Clinical Examination (OSCE) with simulated patient and peer feedback via videoconference}

\author{
Véronique MEISTER ${ }^{1}$, Noëlle JUNOD PERRON ${ }^{2}$, Frédéric GEOFFROY ${ }^{2}$, et Adeline PAIGNON ${ }^{3, *}$ \\ ${ }^{1}$ Programmes compétences cliniques et patients simulés, Faculté de médecine, Université de Genève et Centre interprofessionnel \\ de simulation, Genève, Suisse \\ ${ }^{2}$ Unité de développement et de recherche en éducation médicale (UDREM), Faculté de médecine, Université de Genève et \\ Institut de médecine de premier recours, Hôpitaux universitaires de Genève (HUG), Genève, Suisse \\ ${ }^{3}$ Haute école de santé de Genève, HES-SO Haute école spécialisée de Suisse Occidentale et Centre interprofessionnel de \\ Simulation, Genève, Suisse
}

Manuscrit reçu le 28 avril 2020 ; accepté pour publication le 11 juin 2020

\section{Contexte}

Depuis une quinzaine d'années, le programme des compétences cliniques de la Faculté de médecine de Genève, en lien avec l'Unité de recherche et de développement en éducation médicale (UDREM) développe des stations formatives sous forme d'examens cliniques objectifs et structurés (ECOS) pour les étudiants en médecine des années précliniques (Bachelor 2 et 3). Depuis 2014, le Centre interprofessionnel de simulation (CIS) contribue à la pérennité de ces stations formatives $(\mathrm{SF})$ en adaptant régulièrement les scénarios, en sélectionnant, formant et supervisant les patients simulés (PS) et en assurant la logistique de déploiement des stations.

Lors des SF avec PS, les étudiants s'entraînent à mener une consultation médicale (anamnèse, examen physique, communication avec le patient, raisonnement clinique) et aux transmissions écrites sous forme de rapport de consultation. Les étudiants bénéficient de six stations formatives dont quatre avec PS, une avec un patient instructeur et une consacrée à la présentation orale de cas à un superviseur. Les quatre premières stations se déroulent sous la supervision d'un médecin clinicien généraliste ou spécialiste formé au feedback [1] (pour le détail, voir: https://www.unige.ch/medecine/ fr/enseignement1/bachelor-et-master-en-medecinehumaine/programmeslongitudinaux/competencesclini ques/ccstationsformatives/). Chaque station avec PS

\footnotetext{
${ }^{*}$ Correspondance et offprints: Adeline PAIGNON. Centre Interprofessionnel de Simulation et Haute École de santé de Genève CMU 1, rue Michel Servet, 1211 Genève 4, Suisse. Mailto: adeline.paignon@hesge.ch.
}

aborde un thème en rapport et en continuité avec le cursus théorique selon des objectifs spécifiques. Pour la deuxième station formative (SF2) présentée ici, les étudiants doivent caractériser une douleur thoracique ou faire l'anamnèse d'une insuffisance cardiaque, pratiquer un examen physique ciblé sur le système cardio-vasculaire, construire la relation avec le patient (empathie, légitimation et soutien) et la structurer pendant la consultation.

En temps normal, cette SF2 se déroule selon trois formats différents: supervision directe, vidéo et cabinet médical. Chaque format permet un type de supervision spécifique: feedback court et immédiat pour le format en observation directe, auto-évaluation et feedback différé pour le format en vidéo, feedback participatif avec des pairs et en immersion pour le format en cabinet médical [2].

Dans les trois formats, le PS donne un feedback sur ses ressentis et les aspects relationnels. Après sa station, chaque étudiant note ses points forts et à améliorer ainsi que ses objectifs d'apprentissage pour la future station dans son portfolio électronique GPS (Geneva Portfolio Support). Il y rédige aussi son rapport de consultation. Les rapports de consultations sont travaillés jusqu'à la fin des années précliniques afin de préparer les étudiants à la présentation de cas.

\section{Problématique}

Le 16 mars 2020, l'Université de Genève a fermé ses portes suite aux directives de confinement généralisé édictées par le Conseil Fédéral afin de ralentir la pandémie 
Covid-19. Les étudiants en médecine ont vu leurs cours, séminaires et examens en présence annulés, notamment les $\mathrm{SF}$ avec PS, ces derniers étant des personnes à risques. La SF2 étant déjà planifiée du 26 mars au 12 mai dans les trois formats, il restait à peine 10 jours pour mettre en œuvre une alternative en tenant compte des obligations sanitaires. De plus, les médecins chargés d'enseignement, cliniciens généralistes et spécialistes, ayant été largement mobilisés pour faire face aux besoins en soins de la population, leur disponibilité pour l'enseignement est devenue aléatoire, voire impossible.

Le défi était donc de condenser les trois différents formats habituels en un format de station à distance permettant à 158 étudiants d'exercer les compétences cliniques requises.

\section{Ce que nous avons fait}

L'option choisie a été de combiner le format en cabinet médical et d'observation directe pour créer une nouvelle modalité à distance. Elle devait permettre de réunir deux étudiants, un superviseur et un patient simulé. Afin de respecter le planning, les 158 étudiants ont été répartis en séances d'une heure trente sur les 40 demi-journées de la période initialement prévue. Ainsi, chaque étudiant pouvait bénéficier de 20 minutes d'interaction avec le PS et de 10 à 20 minutes de briefing et de feedback. Afin de pallier le manque de disponibilité des superviseurs médecins, une alternative a été trouvée auprès de 11 étudiants moniteurs de Master 1 et 2, déjà formés par des cliniciens à l'entraînement aux compétences cliniques de leurs cadets.

\section{Étapes préparatoires aux stations formatives à distance}

La mise en œuvre de cette nouvelle SF de type ECOS à distance a nécessité de retravailler les rôles avec les PS, de modifier les consignes de présentation de la SF pour les étudiants et de former les étudiants moniteurs à ce nouveau format.

Ainsi, la sélection et la formation des PS a nécessité de prioriser des PS expérimentés et relativement à l'aise avec l'ordinateur. Quatre se sont lancés dans l'aventure, ils ont été formés à leurs deux rôles (angor stable, patient qui banalise et insuffisance cardiaque, patient qui a stoppé son traitement) lors de deux séances de deux heures en visioconférence. La documentation concernant les scénarios pour les patients simulés n'a pas nécessité de modifications.

Pour les étudiants, seule la consigne de départ a été adaptée avec l'indication de décrire à haute voix les gestes de l'examen clinique et leurs buts. Les objectifs d'entraînement aux compétences cliniques n'ont pas été modifiés.

Pour les étudiants moniteurs, engagés sur la base du volontariat, plusieurs guides ont été rédigés : objectifs et contexte de la station, utilisation de l'outil de visioconférence, utilisation de la grille d'observation et du portfolio électronique de l'étudiant. Leur formation à l'entraînement aux compétences clinique a été complétée par une séance de formation sur les principes et stratégies d'un feedback efficace et constructif. Un guide sur le feedback en SF leur a aussi été remis.

\section{Déroulement d'une séance}

Chacune des séances se fonde sur le même format. L'étudiant moniteur invite par e-mail les étudiants et le patient simulé à la visioconférence. S'il le souhaite, il peut aussi inviter un superviseur expérimenté afin d'avoir un retour sur ses feedbacks. Une fois tous les participants connectés, l'étudiant moniteur procède au briefing et donne des consignes d'observation de la station à l'étudiant observateur. Le premier étudiant effectue sa consultation avec le PS, l'étudiant moniteur observe l'interaction et remplit électroniquement la grille d'observation. L'étudiant observateur relève les points spécifiques selon les consignes d'observation. À la fin de la consultation, le feedback est effectué entre l'étudiant moniteur, les deux étudiants et le PS et les éléments principaux sont consignés dans le portfolio. Le même procédé est ensuite utilisé avec le deuxième étudiant ; dans ce cas le PS joue un rôle différent mais la grille d'observation reste la même. À la fin de la séance, l'étudiant moniteur valide les objectifs généraux à atteindre sur le portfolio de chaque étudiant par partage d'écran. Hors ligne, chaque étudiant complète le rapport de consultation qu'il soumet à son binôme pour évaluation.

\section{Ce que nous avons observé}

$\mathrm{Au}$ vu des séances réalisées, il s'avère que ce type de modalité en ligne répond de manière efficace aux contraintes imposées par les directives de confinement sans altérer le déroulement de la formation des étudiants en Bachelor 2.

Tout d'abord, l'ensemble des étudiants de Bachelor 2 a accueilli favorablement ce format inédit d'enseignement à distance. Ils n'ont pas rencontré de difficulté dans l'utilisation de la visioconférence et se sont immergés rapidement dans la situation clinique. Ils ont ainsi pu s'exercer aux compétences cliniques et atteindre les objectifs prévus pour ce qui concerne l'anamnèse et la communication avec le patient. Malgré l'impossibilité de pratiquer l'examen physique la visualisation verbalisée du déroulement de l'examen clinique peut cependant permettre son intégration.

Cette modalité à distance donne aussi l'opportunité aux étudiants moniteurs de réviser des compétences cliniques déjà acquises au cours de leur propre formation, ainsi que de développer leurs compétences pédagogiques. Cet entraînement à la supervision d'étudiants lors d'examen clinique leur donne l'occasion d'exercer certains aspects de leur futur rôle en tant que médecin. 
L'interaction bienveillante avec les étudiants moniteurs a permis aux PS de jouer leurs rôles sans difficulté et de proposer des feedbacks en adéquation avec les objectifs d'apprentissage de la station. Ils nous ont aussi fait part de leur intérêt certain à participer à ce nouveau format.

La visioconférence semble être un moyen simple pour introduire dans le cursus un nouveau format consacré à des exercices spécifiques, mais répond mal au besoin d'intégration des savoir-faire et des savoir-être durant l'examen clinique. Un complément pour combler cette lacune devra être envisagé après la période de confinement.

Les effets et implications de cette SF en ligne consacrée à l'apprentissage des compétences cliniques restent encore à évaluer.

\section{Conclusion}

Dans ce contexte de confinement, l'opportunité s'est présentée d'adapter le dispositif complexe des SF de type ECOS en un nouveau format à distance. Grâce à la motivation et à l'énergie de chaque personne impliquée dans ce projet, 158 étudiants en médecine de Bachelor 2 peuvent s'exercer aux compétences cliniques malgré le confinement. De plus, 11 étudiants de Master 1 et 2 ont bénéficié de l'opportunité de pratiquer la supervision et de développer ainsi leurs compétences pédagogiques. Enfin, malgré l'absence de contact réel avec le patient, ce format de SF par visioconférence permet aux étudiants apprenants et moniteurs d'expérimenter la télémédecine qui fera certainement partie de leur réalité dans un futur proche [3].

\section{Références}

1. Junod Perron N, Louis-Simonet M, Cerutti B, Pfarrwaller E, Sommer J, Nendaz M. Feedback in formative OSCEs: Comparison between direct observation and video-based formats. Med Educ Online 2016;21:32160.

2. Junod Perron N, Louis-Simonet M, Cerutti B, Pfarrwaller E, Sommer J, Nendaz M. The quality of feedback during formative OSCEs depends on the tutors' profile. BMC Med Educ 2016;16:293.

3. McCoy CE, Sayegh J, Alrabah R, Yarris LM. Telesimulation: An innovative tool for health professions education. AEM Education and Training 2017;1:132-6.

Citation de l'article : Meister V., Junod Perron N., Geoffroy F., Paignon A. Examen clinique objectif structuré (ECOS) formatif avec patient simulé et rétro-action par un pair en visioconférence. Pédagogie Médicale 2020:21;219-221 